Reserch Article

\title{
Comparison of Post-mortem 7.0-Tesla Magnetic Resonance Imaging of the Brains of Alzheimer Patients with and without Cerebral Amyloid Angiopathy
}

Jacques De Reuck *, Florent Auger, Nicolas Durieux, Claude-Alain Maurage, Vincent Deramecourt, Charlotte Cordonnier, Florence Pasquier, Didier Leys, Regis Bordet

Unitè 1171 "Degenerative and vascular cognitive disorders", Université de Lille 2, Lille, France; EMails: dereuck.j@gmail.com; florent.auger@inserm.fr; nicolas.durieux@univ-lille2.fr; ClaudeAlain.MAURAGE@CHRU-LILLE.FR; vderamecourt@aol.com; Charlotte.CORDONNIER@CHRULILLE.FR; Florence.PASQUIER@CHRU-LILLE.FR; Didier.LEYS@CHRU-LILLE.FR; Regis.BORDET@CHRULILLE.FR.

* Correspondence: Jacques De Reuck; E-Mail: dereuck.j@gmail.com

Academic Editor: James S. Powers

Special Issue: Cerebrovascular Biomarkers in Alzheimer's Disease

OBM Geriatrics

2020, volume 4, issue 4

doi:10.21926/obm.geriatr.2004142
Received: September 20, 2020

Accepted: November 16, 2020

Published: December 10, 2020

\begin{abstract}
Purpose: The influence of cerebral amyloid angiopathy (CAA) in Alzheimer's disease (AD) remains unexplored. The present post-mortem study investigated possible differences in the degree of hippocampal atrophy $(\mathrm{HA})$ between $A D$ patients with and without CAA using 7.0tesla magnetic resonance imaging (MRI). Also, the incidence of the hippocampal cortical micro-infarcts (HCoMIs) and hippocampal cortical micro-bleeds (HCoMBs) is compared to those in the neocortex. Methods: The examined post-mortem brains included 30 AD-CAA cases and $20 \mathrm{AD}$ without CAA cases. The samples of the hippocampus were evaluated on the most representative coronal section with $\mathrm{T} 2$ and $\mathrm{T} 2 * \mathrm{MRI}$ sequences. The average degree of $\mathrm{HA}$ was determined in both groups. The incidences of HCoMls and HCoMBs, along with the frequency of CoMls and CoMBs in the neocortex were compared in both groups: $A D$-with CAA and AD without CAA cases. Results: No significant differences were observed in the degree of
\end{abstract}

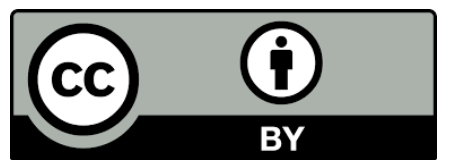

(C) 2020 by the author. This is an open access article distributed under the conditions of the Creative Commons by Attribution License, which permits unrestricted use, distribution, and reproduction in any medium or format, provided the original work is correctly cited. 
$\mathrm{HA}$ and the incidence of hippocampal micro-infarcts (HMIs) and hippocampal micro-bleeds (HMBs) between the AD-CAA and the AD brains in contrast to the higher incidence of these cerebrovascular lesions in the neocortex of AD-CAA brains. The incidence of CoMIs and CoMBs in the neocortex showed similarity to that in the hippocampus of AD patients without CAA. Conclusions: CAA does not influence the degree of $\mathrm{HA}$ and the incidence of micro-infarcts (MIs) and micro-bleeds (MBs) in the hippocampus, in contrast to the high contribution of the latter with CAA in the neocortex. The hippocampus seems to be more spared from cerebrovascular involvement than the other parts of the brain.

\section{Keywords}

Post-mortem 7.0-tesla magnetic resonance imaging; Alzheimer's disease; cerebral amyloid angiopathy; Hippocampal atrophy; cortical micro-infarcts; cortical micro-bleeds

\section{Introduction}

No previous post-mortem study has established a clear association between the presence of $\beta$ amyloid and the rates of hippocampal atrophy (HA) in patients with Alzheimer's disease (AD). In a study, TAR DNA-binding protein 43 (TDP-43) was considered as a potential factor/marker related to increased rates of HA in AD patients [1]. Several studies have established that the rate of HA is associated with both baseline $\beta$-amyloid load and accumulation [2]. Also, several arterioles exhibiting significant alpha-smooth muscle actin loss were observed in the hippocampus and the gyrus frontalis medialis in brains of AD patients, which was related to the degree of cerebral amyloid angiopathy (CAA) [3]. Also, capillary CAA and CAA severity were found to be associated with HMIs [4].

Resultantly, divergent opinions concerning the impact of CAA on the degree of HA and the degree of associated cerebrovascular lesions in AD patients have been documented.

The present post-mortem 7.0-tesla magnetic resonance imaging (MRI) study compares the degree of HA between $A D$ brains with associated severe CAA and those without CAA. Also, the incidences of the HMIs and HMBs between $A D-C A A$ and $A D$ brains are compared. Also, their incidence is compared to the frequency of cortical micro-infarcts (CoMIs) and cortical micro-bleeds (CoMBs) in the neocortex.

\section{Materials and Methods}

Thirty $A D-C A A$ and $20 A D$ brains in post-mortem were investigated. A previously obtained informed consent of the patients or from the closest family member allowed an autopsy for diagnostic and scientific purposes. The brain tissue samples were acquired from the Lille NeuroBank of the Lille University, France that is part of the "Centre de Ressources Biologiques" and acts as an institutional review board (IRB).

The neuropathological examination was conducted according to a standard procedure [5]. The Braak criteria were employed to determine the severity of the tau pathology. Only grade $\mathrm{V}$ and $\mathrm{VI}$ stages were retained as a definitive diagnosis of $A D[6]$. The presence of various degrees of CAA was 
made according to the criteria of a consensus protocol with gradations from 0 to 3 on examining cortical samples of the frontal, temporal, parietal, and occipital cortex with $\beta$-amyloid staining [7].

A 7.0-Tesla MRI Bruker BioSpin SA (BioSpin GmbH, Ettlingen, Germany) was used with an issuerreceiver cylinder coil with an inner diameter of $72 \mathrm{~mm}$. The same was used in an earlier study [8]. Three to six coronal sections of a cerebral hemisphere were submitted to SPIN ECHO T2 and T2* MRI sequences before the brain sampling for the histological examination. The hippocampus was evaluated on the most representative section. The degree of in vivo HA was determined using Scheltens criteria $[9,10]$. This criterion was applied to post-mortem 7.0-tesla MRI [11]. Also, the incidence of HMIs and HMBs were evaluated for cortical hemispheric CoMIs and CoMBs [12].

Uniform comparisons of unpaired groups were made with Fisher's exact test for categorical data. The non-parametric Mann-Whitney U-test was employed to compare continuous variables. The significance level, two-tailed, was set at $\leq 0.01$ for significant and $\leq 0.001$ for highly significant. $p$ Values set at $\leq 0.05$ and more than $>0.01$ were considered as marginally significant.

\section{Results}

The mean ages of the patients with AD-CAA and with AD were almost similar and were 77 (6384) years, and 78 (65-83) years, respectively. No gender differences were observed with an incidence of $41 \%$ in the AD-CAA brains and $40 \%$ in the $A D$ ones. A territorial infarct was noticed in five $(25 \%)$ AD-CAA brains and in one (3\%) patient with AD without CAA. Lobar hematomas were found in eight (40\%) AD-CAA brains and were restricted to one (3\%) with only the AD.

No differences concerning the degree of $\mathrm{HA}$ and the incidence of HMIs and HMBs between the $A D$ and the $A D-C A A$ brains were observed. A significant difference in the occurrence of CoMls and CoMBs was found in the neocortex with a considerable increase in the AD-CAA brains $(p \leq 0.001)$, compared to the similar rate of HMIs and HMBs in the AD and AD-CAA ones (Figure 1 and Figure 2). The occurrence of HMIs and HMBs was more or less similar as in the neocortex of "pure" AD patients (Table 1).

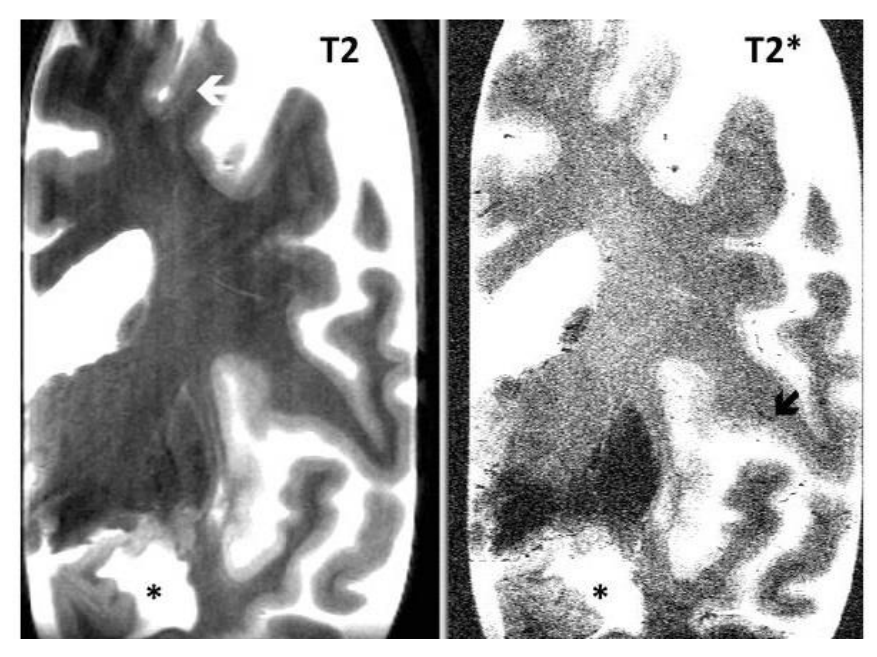

Figure $1 \mathrm{~T} 2$ and $\mathrm{T} 2 *$ sequences of a coronal section of a cerebral hemisphere in a patient with $A D$ and CAA. Note in addition to the severe dilation of the temporal horn and the small hippocampus $(*)$, a cortical micro-infarct (white arrow) on the T2 sequence and two cortical micro-bleeds (black arrow) on the T2*. 


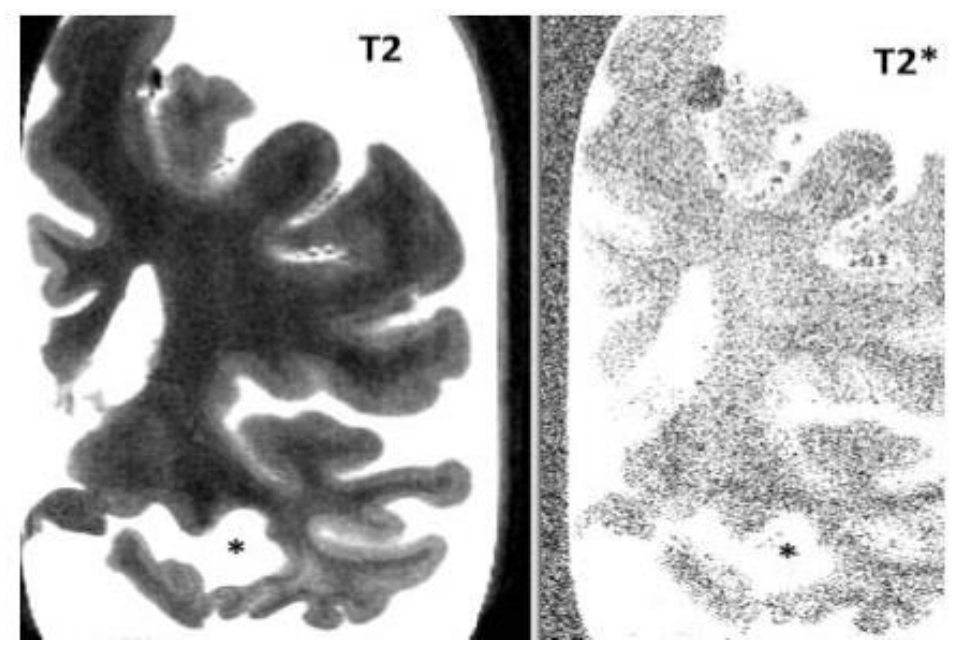

Figure $2 \mathrm{~T} 2$ and $\mathrm{T} 2 *$ sequences of a coronal section of a cerebral hemisphere in a patient with Alzheimer's dementia without cerebral amyloid angiopathy. There is severe dilation of the temporal horn with a small remaining hippocampus $\left({ }^{*}\right)$.

Table 1 Comparison of the degree of hippocampal lesions in Alzheimer's disease (AD) with and without cerebral amyloid angiopathy (CAA) and the incidence of cerebrovascular lesions in the neocortex.

\begin{tabular}{ccccc}
\hline \multirow{2}{*}{ Items } & \multicolumn{2}{c}{ Hippocampus } & \multicolumn{2}{c}{ Neocortex } \\
\cline { 2 - 5 } & AD-CAA & AD & AD-CAA & AD \\
\hline Hippocampal atrophy & $2.7(1.1)$ & $2.6(1.2)$ & & \\
Cortical micro-infarcts & $0.2(0.4)$ & $0.1(0.4)$ & $2.1(2.2)^{* * *}$ & $0.1(0.2)$ \\
Cortical micro-bleeds & $0.7(0.9)$ & $0.5(0.8)$ & $1.7(1.2)^{* * *}$ & $0.5(0.7)$ \\
\hline \multicolumn{7}{c}{$\mathrm{P}^{* * *}: \leq 0.001$} &
\end{tabular}

\section{Discussion}

There is a good correlation between the visual MRI quantification of medial temporal lobe atrophy and HA compared to the classical neuropathological evaluation [13].

The present study revealed no difference in the degree of HA and the frequency of HMIs and HMBs between AD-CAA brains and AD brains. This is in contrast with the high incidence of neocortical CoMls and CoMBs in AD-CAA brains compared to "pure" AD ones ( $\leq 0.001)$. In the present post-mortem study, no association between $\beta$-amyloid or tau proteins and rates of HA was established in $A D$ brains.

$H A$ is not specific to $A D$ with or without CAA but is also observed in frontotemporal dementia [14, 15]. The higher incidence of neocortical CoMils and CoMiBs has already been described in some earlier studies [16, 17].

Both $A D-C A A$ and $A D$ patients were of similarly advanced age when they died. The disease duration was not evaluated, as the early clinical onset of $A D$ is not always clear. During the aging process, only increased severity of white matter changes and the incidence of CoMBs are observed. CoMls showed no increase with advancing age [18]. 
Some previous studies have shown a higher incidence of CoMls and CoMiBs in the neocortex of patients with AD-CAA compared to AD ones [12, 19, 20]. Also, in CAA patients without AD features, the incidence of these lesions is even higher than in AD-CAA ones [21].

\section{Conclusion}

It can be concluded that CAA has no significant influence on the degree of HA and the associated small cerebrovascular lesions in contrast to those in the hemispheric neocortex. Further studies on large populations are suggested to establish a clear link and thereby take proper diagnostic and preventive interventions to reduce morbidities from the deadly disease of CAA.

\section{Author Contributions}

Jacques De Reuck has designed the study. Together with Florent Auger and Nicolas Durieux he performed the MRI examinations. Claude-Alain Maurage and Vincent Deramecourt performed the macroscopic and histological examinations of the brains. Charlotte Cordonnier, Florence Pasquier, Didier Leys and Regis Bordet were responsible for clinical evaluation during life.

\section{Competing Interests}

The authors have declared that no competing interests exist.

\section{References}

1. Joseph KA, Dickson DW, Tosakulwong N, Weigand SD, Murray ME, Petrucelli L, et al. Rates of hippocampal atrophy and presence of post-mortem TDP-43 in patients with Alzheimer's disease: A longitudinal retrospective study. Lancet Neurol. 2017; 16: 917-924.

2. Andrews KA, Frost C, Modat M, Cardaso MJ, Rowe CC, Villemagne V, et al. Acceleration of hippocampal atrophy rates in asymptomatic amyloidosis. Neurobiol Aging. 2016; 39: 99-107.

3. Merlini M, Wanner D, Nitsch RM. Tau pathology-dependent remodelling of cerebral arteries precedes Alzheimer's disease-related microvasular cerebral amyloid angiopathy. Acta Neuropathol. 2016; 131: 737-752.

4. Hecht M, Krämer LM, von Arnim CA, Otto M, Thal DR. Capillary cerebral amyloid angiopathy in Alzheimer's disease association with allocortex: Hippocampal microinfarcts and cognitive decline. Acta Neuropathol. 2018; 135: 681-694.

5. De Reuck J. Histopathological stai nings and definitions of vascular disruptions in the elderly brain. Exp Geront. 2012; 47: 834-837.

6. Braak H, Braak E. Neuropathological staging of Alzheimer-related changes. Acta Neuropathol. 1991; 82: 239-259.

7. Love S, Chalmers K, Ince P, Esiri M, Attems J, Jellinger K, et al. Development, appraisal, validation and implementation of a consensus protocol for assessment of cerebral amyloidal angiopathy in post-mortem brain tissue. Am J Neurodegener Dis. 2014; 3: 19-32.

8. De Reuck J, Auger F, Cordonnier C, Deramecourt V, Durieux N, Pasquier F, et al. Comparison of 7.0-T T2*-magnetic resonance imaging of cerebral bleeds in post-mortem brain sections of Alzheimer patients with their neuropathological correlates. Cerebrovasc Dis. 2011; 31: 511-517. 
9. Scheltens $P$, Leys $D$, Barkhof $F$, Huglo D, Weinstein HC, Vermersch $P$, et al. Atrophy of medial temporal lobes on MRI in "probable" Alzheimer's disease and normal aging: Diagnostic value and neuropsychological correlates. J Neurol Neurosurg Psychiatry. 1992; 55: 967-972.

10. Wahlund $L$, Julin $P$, Johansson $S$, Scheltens $P$. Visual rating and volumetry of the medial temporal lobe on magnetic resonance imaging: A comparative study. J Neurol Neurosurg Psychiatry. 2000; 69: 630-635.

11. Blanken AE, Hurtz S, Zarow C, Biado K, Honarpisheh H, Somme J, et al. Association between hippocampal morphometry and neuropathological markers of Alzheimer's disease using $7 \mathrm{~T}$ MRI. Neuroimage Clin. 2017; 15: 56-61.

12. De Reuck J, Auger F, Durieux N, Deramecourt V, Maurage CA, Pasquier F, et al. Cerebrovascular lesions during normal aging: A neuropathological study with 7.0-tesla magnetic resonance imaging. EC Neurology. 2018; 10: 229-235.

13. Boutet $C$, Chupin M, Colliot $O$, Sarazin M, Mutlu G, Drier A, et al. Is radiological evaluation as good as computer-based volumetry to assess hippocampal atrophy in Alzheimer's disease? Neuroradiology. 2012; 54: 1321-1330.

14. de Souza LC, Chupin M, Bertoux M, Lehéricy S, Dubois B, Lamari F, et al. Is hippocampal volume a good marker to differentiate Alzheimer's disease from frontotemporal dementia? J Alzheimers Dis. 2013; 36: 57-66.

15. Hornberger $\mathrm{M}$, Wong $\mathrm{S}$, Tan $\mathrm{R}$, Irish $\mathrm{M}$, Piguet $\mathrm{O}$, Kril J, et al. In vivo and post-mortem memory circuit integrity in frontotemporal dementia and Alzheimer's disease. Brain. 2012; 135: 30153025.

16. De Reuck J, Auger F, Durieux N, Maurage CA, Deramecourt V, Cordonnier C, et al. Post-Mortem 7.0-tesla magnetic resonance imaging of the hippocampus during normal aging and in neurodegenerative dementias. SunText Rev Neurosci Psychol. 2020; 1: 108-113.

17. De Reuck J, Deramecourt V, Auger F, Durieux N, Cordonnier C, Devos D, et al. Post-mortem 7.0tesla magnetic resonance study of cortical micro-infarcts in neurodegenerative diseases and vascular dementia with neuropathological correlates. J Neurol Sci. 2014; 346: 85-89.

18. De Reuck J, Cordonnier C, Deramecourt V, Auger F, Durieux N, Bordet R, et al. Microbleeds in postmortem brains of patients with Alzheimer disease. A T2*-weighted gradient-echo $7.0 \mathrm{~T}$ magnetic resonance imaging study. Alzheimer Dis Assoc Disord. 2013; 27: 162-167.

19. Kimberly WT, Gilson A, Rost NS, Rosand J, Viswanathan A, Smith EE, Greenberg SM. Silent ischemic infarcts are associated with hemorrhage burden in cerebral amyloid angiopathy. Neurology. 2009; 72: 1230-1235.

20. van Rooden S, Goos JD, van Opstal AM, Versluis MJ, Webb AG, Blauw GJ, et al. Increased number of microinfarcts in Alzheimer disease at 7-T MR imaging. Radiology. 2014; 270: 205-211.

21. De Reuck J, Cordonnier C, Auger F, Durieux N, Maurage CA, Deramecourt V, et al. Cerebrovascular lesions in cerebral amyloid angiopathy with and without Alzheimer's disease: A neuropathological study with post-mortem 7.0-t magnetic resonance imaging. EC Neurology. 2018; 10: 954-960. 


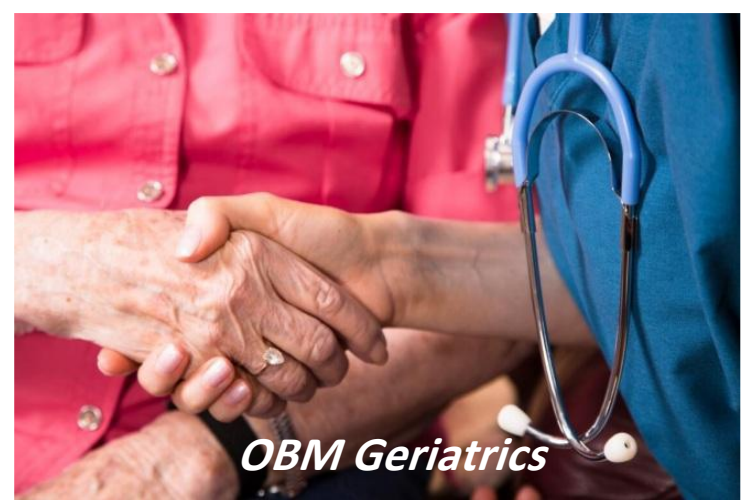

Enjoy OBM Geriatrics by:

1. Submitting a manuscript

2. Joining in volunteer reviewer bank

3. Joining Editorial Board

4. Guest editing a special issue

For more details, please visit: http://www.lidsen.com/journals/geriatrics 